\title{
Distinguishing Probability Weighting from Risk Misperceptions in Field Data
}

Levon Barseghyan

Cornell University

Francesca Molinari

Cornell University

Ted O'Donoghue

Cornell University

Joshua C. Teitelbaum

Georgetown University Law Center, jct48@law.georgetown.edu

Georgetown Business, Economics and Regulatory Law Research Paper No. 13-002

doi:http://www.aeaweb.org/articles.php?doi=10.1257/aer.103.3.580

This paper can be downloaded free of charge from:

https://scholarship.law.georgetown.edu/facpub/1155

http://ssrn.com/abstract=2200543

103 Am. Econ. Rev. Papers \& Proceedings 580-585 (2013)

This open-access article is brought to you by the Georgetown Law Library. Posted with permission of the author. Follow this and additional works at: https://scholarship.law.georgetown.edu/facpub

Part of the Behavioral Economics Commons, Insurance Law Commons, and the Law and Economics Commons 


\title{
Distinguishing Probability Weighting from Risk Misperceptions in Field Data
}

\author{
By Levon Barseghyan, Francesca Molinari, Ted O’Donoghue, \\ and Joshua C. Teitelbaum*
}

There is a large literature that attempts to estimate risk preferences from field data (e.g., Jullien and Salanié 2000 (horse race bets); Cohen and Einav 2007 (auto insurance); Snowberg and Wolfers 2010 (horse race bets); Sydnor 2010 (home insurance); Barseghyan et al. forthcoming (auto and home insurance)). Many of the studies in this literature consider nonstandard models of risk preferences. One particular model that has received a great deal of attention is "probability weighting."

The idea of probability weighting is that agents behave roughly as in expected utility theory, except that they evaluate probabilities nonlinearly, transforming them into decision weights. Probability weighting was popularized by Kahneman and Tversky (1979) as part of prospect theory. Their original approach, however, can lead to violations of stochastic dominance, and so the model of probability weighting typically used today incorporates rank dependence as proposed by Quiggin (1982). ${ }^{1}$ Under rank-dependent probability weighting (RDPW), agents transform cumulative instead of individual probabilities, and consequently the decision weights depend on the ranking of outcomes. The RDPW model has been studied extensively in

\footnotetext{
* Barseghyan: Department of Economics, Cornell University, Ithaca, NY 14853 (e-mail: 1b247@cornell.edu); Molinari: Department of Economics, Cornell University, Ithaca, NY 14853 (e-mail: fm72@ cornell.edu); O'Donoghue: Department of Economics, Cornell University, Ithaca, NY 14853 (e-mail: edo1@ cornell.edu); Teitelbaum: Georgetown University Law Center, 600 New Jersey Avenue NW, Washington, DC 20001 (e-mail: jct48@ law.georgetown.edu). We thank Ben Handel and the other session participants, Nick Barberis, Ori Heffetz, and seminar participants at Berkeley. We acknowledge financial support from NSF grants SES1031136 and SES-092230 (Molinari).

${ }^{\dagger}$ To view additional materials, and author disclosure statement(s),visit the article page at http://dx.doi.org/10.1257/aer.103.3.580.

${ }^{1}$ Cumulative prospect theory (Tversky and Kahneman 1992) incorporates Quiggin's notion of rank dependence.
}

laboratory experiments, producing a large body of evidence in support of the theory (for a recent review, see Fehr-Duda and Epper 2012).

When economists take RDPW models to field data, however, an important confound emerges: systematic risk misperceptions (RM). In the laboratory, when subjects are explicitly told that an event occurs with probability 3 percent, and then behave as if the event occurs with probability 7 percent, this behavior clearly is not driven by RM. In the field, by contrast, when researchers assess that an event occurs with probability 3 percent, but agents behave as if the event occurs with probability 7 percent, this behavior could be driven by RM. Indeed, recent papers that estimate RDPW models using field data explicitly acknowledge this issue (e.g., Snowberg and Wolfers 2010; Sydnor 2010; Barseghyan et al. forthcoming) ${ }^{2}$

In this paper, we outline a strategy for distinguishing RDPW from RM in field data. Our strategy relies on identifying a field environment with two key properties (which are not satisfied in most existing studies): (i) the objects of choice are money lotteries with more than two outcomes and (ii) the ranking of outcomes differs across lotteries. In such environments, the ranking of outcomes is irrelevant to agents' decision weights under RM, which simply correspond to their misperceived probabilities, but it is crucial to agents' decision weights under RDPW, which are rank dependent. Thus, the models can make distinct predictions and thereby can be distinguished empirically (although exactly how their predictions differ depends on the details of the specific environment). In the remainder of the paper, we elucidate the general point and illustrate the details within one particular application using simulated data.

\footnotetext{
${ }^{2}$ Barberis (2013) also highlights this issue.
} 


\section{The Model}

An agent faces a choice among money lotteries. Under each lottery $\ell$, the agent pays a price $p(\ell)$ and faces $N$ potential "income" events, which represent gains or losses to wealth. An income event $n$ occurs with probability $\mu_{n}$ and generates income $x_{n}(\ell)$, where either $x_{n}(\ell)<0$ or $x_{n}(\ell)>0$ for all $\ell$ and $n=1, \ldots, N$. With probability $1-\mu_{1}-\cdots-\mu_{N}$ the agent does not experience an income event. Thus, we can write

$$
\begin{aligned}
\ell \equiv & -p(\ell)+x_{1}(\ell), \mu_{1} ; \ldots ; \\
& \left.-p(\ell)+x_{N}(\ell), \mu_{N} ;-p(\ell), 1-\sum_{n=1}^{N} \mu_{n}\right) .
\end{aligned}
$$

This structure can suit many applications. For example, it can capture deductible choices in insurance, where $p(\ell)$ is the total premium paid for insurance against $N$ losses, and $x_{n}(\ell)<0$ is the deductible paid if loss $n$ occurs. Similarly, it can describe bets on horse races, where $p(\ell)$ is the aggregate wager on $N$ bets, and $x_{n}(\ell)>0$ is the payoff received if bet $n$ pays. ${ }^{3}$

Without loss of generality, take a lottery $\ell$ such that $x_{1}(\ell) \leq \cdots \leq x_{N}(\ell)<0$. Under expected utility with correct beliefs, the utility of $\ell$ is given by

$$
\begin{aligned}
U(\ell)= & \sum_{n=1}^{N} \mu_{n} u\left(w-p(\ell)+x_{n}(\ell)\right) \\
& +\left[1-\sum_{n=1}^{N} \mu_{n}\right] u(w-p(\ell)) .
\end{aligned}
$$

The function $u(\cdot)$ is a utility function defined over final wealth, and $w$ is initial wealth.

A possible deviation from (1) is a RM model. A second possible deviation is a RDPW model. Under both models, the utility of $\ell$ is given by

$$
\begin{aligned}
U(\ell)= & \sum_{n=1}^{N} \omega_{n} u\left(w-p(\ell)+x_{n}(\ell)\right) \\
& +\left[1-\sum_{n=1}^{N} \omega_{n}\right] u(w-p(\ell)) .
\end{aligned}
$$

\footnotetext{
${ }^{3}$ Note that under this structure $\mu_{n}$ is independent of $\ell$. This holds in the case of horse bets, where the bets do not influence the probability of winning (unless the race is fixed). In the case of insurance deductibles, it is a reasonable approximation provided that any deductible-related moral hazard is small.
}

The two models, however, differ on the decision weights, $\omega_{1}, \ldots, \omega_{N}$. Under RM, the weight on income event $n$ is $\omega_{n}=\psi\left(\mu_{n}\right)$ for all $n$, where $\psi\left(\mu_{n}\right)$ is the agent's incorrect belief regarding the probability of event $n$. Under RDPW, by contrast, the weights are $\omega_{1}=\pi\left(\mu_{1}\right)$ and $\omega_{n}=\pi\left(\sum_{i=1}^{n} \mu_{i}\right)$ - $\pi\left(\sum_{i=1}^{n-1} \mu_{i}\right)$ for $n \geq 2$, where $\pi(\cdot)$ is a probability weighting function..$^{4}$ Consequently, except for the special case where $\pi(\mu)=c \mu$ for some $c>0$, the decision weights depend on the ranking of outcomes under RDPW but are independent of the ranking of outcomes under RM. It follows that if the ranking of outcomes differs across lotteries, the decision weights differ across lotteries under RDPW but are identical across lotteries under RM.

\section{A. Binary Lotteries}

In most studies that estimate risk preferences from field data, there is one income event that either occurs or does not occur, and for all options in the choice set the payoff when the event occurs is always worse (insurance) or always better (gambling) than the payoff when the event does not occur. In this case, both models are given by

$$
\begin{aligned}
U(\ell)= & \omega_{1} u(w-p(\ell)+x(\ell)) \\
& +\left(1-\omega_{1}\right) u(w-p(\ell)),
\end{aligned}
$$

where $\omega_{1}=\psi\left(\mu_{1}\right)$ under $\mathrm{RM}$ and $\omega_{1}=\pi\left(\mu_{1}\right)$ under RDPW. Because the ranking of outcomes is the same for all lotteries, RDPW loses the distinction that the weights differ across lotteries. Hence, the two models are indistinguishable.

\section{B. Lotteries with Multiple Income Events}

With multiple income events, however, it becomes possible to distinguish RDPW from $\mathrm{RM}$. The key additional requirement is that the ranking of outcomes differs across lotteries. To illustrate the intuition, it suffices to consider the case of two income events and two lotteries.

\footnotetext{
${ }^{4}$ As in Tversky and Kahneman (1992), in the loss domain we order outcomes from largest loss to smallest loss. For the case of bets, which are in the gain domain, Tversky and Kahneman order outcomes from largest gain to smallest gain (i.e., $x_{1}(\ell) \geq \cdots \geq x_{N}(\ell)>0$ ), which yields identical equations for $\omega_{1}, \ldots, \omega_{N}$.
} 
Suppose there are two income events, 1 and 2, and two lotteries, $a$ and $b$. Suppose further that (i) $x_{1}(\ell)<0$ and $x_{2}(\ell)<0$ for $\ell=a, b$ and (ii) $x_{1}(a)<x_{2}(a)$ and $x_{2}(b)<x_{1}(b)$. Under RM, the decision weights do not depend on the ranking of outcomes and, hence, are the same for lotteries $a$ and $b$. In particular, the weights are $\omega_{1}=\psi\left(\mu_{1}\right)$ and $\omega_{2}=\psi\left(\mu_{2}\right)$, whether the agent chooses lottery $a$ or $b$. Under RDPW, by contrast, the decision weights are dependent on the ranking of outcomes and, hence, differ for lotteries $a$ and $b$. In particular, the weights are $\omega_{1}=\pi\left(\mu_{1}\right)$ and $\omega_{2}=\pi\left(\mu_{1}+\mu_{2}\right)-\pi\left(\mu_{1}\right)$ if the agent chooses lottery $a$ but are $\omega_{1}=\pi\left(\mu_{1}+\mu_{2}\right)-\pi\left(\mu_{2}\right)$ and $\omega_{2}=\pi\left(\mu_{2}\right)$ if the agent chooses lottery $b$.

The fact that the decision weights differ across lotteries under RDPW but are identical across lotteries under RM is a testable implication that potentially can be used to distinguish RDPW from RM in this environment. This fact also implies that, with multiple income events and lottery-dependent outcome rankings, the RM and RDPW models can make different predictions. The exact nature of the models' predictions and their differences depend on the specific application. In the next section, we consider the application of deductible choices in insurance.

\section{Application: Insurance Deductible Choices}

\section{A. A Model of Deductible Choice}

Heterogeneous agents purchase insurance against two potential losses. Each agent is characterized by a vector of loss probabilities $\boldsymbol{\mu}=\left(\mu_{1}, \mu_{2}\right)$ and a vector of base premia $\mathbf{q}=\left(q_{1}, q_{2}\right)$. We assume the agent ignores the possibility of incurring both losses. . $^{\text {. }}$

Each agent purchases a separate policy for each loss. Each policy $n$ provides full insurance against loss $n$, subject to a deductible $d_{n}$ chosen by the agent. Under each policy, the agent faces $J>2$ deductible options, the highest of which never exceeds the size of the covered loss. The agent's base premium $q_{n}$, together with its chosen deductible $d_{n}$, determines the premium $p_{n}$ paid by the agent for policy $n$, i.e., $p_{n}\left(d_{n}\right)=f\left(d_{n} ; q_{n}\right)$. Let $\mathbf{d}=\left(d_{1}, d_{2}\right)$ denote the vector of deductibles

\footnotetext{
${ }^{5}$ This assumption is plausible. It is analogous to assuming the agent ignores the possibility of a single loss occurring multiple times. Moreover, it is testable in field data.
}

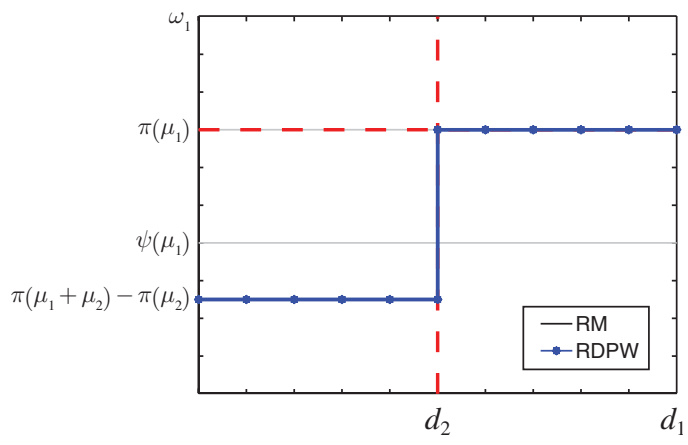

Figure 1. Discontinuity IN WeIGHTS UNDER RDPW

chosen by the agent and $p(\mathbf{d})=p_{1}\left(d_{1}\right)+p_{2}\left(d_{2}\right)$ denote the total premium paid by the agent.

In this environment, each agent faces a choice among $J^{2}$ lotteries of the form

$$
\begin{aligned}
\ell_{\mathbf{d}}= & \left(-p(\mathbf{d})-d_{1}, \mu_{1} ;-p(\mathbf{d})-d_{2}, \mu_{2} ;\right. \\
& \left.-p(\mathbf{d}), 1-\mu_{1}-\mu_{2}\right) .
\end{aligned}
$$

The utility of lottery $\ell_{\mathrm{d}}$ is given by

$$
\begin{aligned}
U\left(\ell_{\mathbf{d}}\right)= & \omega_{1} u\left(w-p(\mathbf{d})-d_{1}\right) \\
& +\omega_{2} u\left(w-p(\mathbf{d})-d_{2}\right) \\
& +\left[1-\omega_{1}-\omega_{2}\right] u(w-p(\mathbf{d})) .
\end{aligned}
$$

Under RM, $\omega_{1}=\psi\left(\mu_{1}\right) \quad$ and $\quad \omega_{2}=\psi\left(\mu_{2}\right)$ for all $\ell_{\mathbf{d}}$. Under RDPW, (i) $\omega_{1}=\pi\left(\mu_{1}\right)$ and $\omega_{2}=\pi\left(\mu_{1}+\mu_{2}\right)-\pi\left(\mu_{1}\right)$, if $d_{1} \geq d_{2}$, or (ii) $\omega_{1}=\pi\left(\mu_{1}+\mu_{2}\right)-\pi\left(\mu_{2}\right)$ and $\omega_{2}=\pi\left(\mu_{2}\right)$, if $d_{1} \leq d_{2}$. As this environment corresponds to the one in Section IB with $N=2$, we know that it may be possible to distinguish RDPW from RM using choice data from this environment.

To develop some intuition for what patterns in the data would select RDPW versus RM, we need to investigate exactly how the RDPW decision weights differ across lotteries. Here, we develop implications of the probability weighting functions typically used in the literature. ${ }^{6}$ A key feature of these functions is that they are subadditive-i.e., $\pi\left(\mu_{1}+\mu_{2}\right)<\pi\left(\mu_{1}\right)+\pi\left(\mu_{2}\right)$-for relatively small probabilities.

\footnotetext{
${ }^{6}$ Take, e.g., the probability weighting functions in Tversky and Kahneman (1992) and Prelec (1998), and the probability distortion function estimated in Barseghyan et al. (forthcoming).
} 
Assuming that $\pi(\cdot)$ is subadditive, Figure 1 depicts for both models how the weight $\omega_{1}$ on loss event 1 varies with $d_{1}$ holding constant $d_{2}$. Under RM, the weight $\omega_{1}$ is the same for all $d_{1}$ (i.e., $\omega_{1}=\psi\left(\mu_{1}\right)$ for all $d_{1}$ ). Under RDPW, however, the weight $\omega_{1}$ is small for $d_{1} \leq d_{2}$ (when $\omega_{1}=$ $\left.\pi\left(\mu_{1}+\mu_{2}\right)-\pi\left(\mu_{2}\right)\right)$ and large for $d_{1} \geq d_{2}$ (when $\left.\omega_{1}=\pi\left(\mu_{1}\right)\right)$. This discontinuity creates "kinks" in the utility function that encourage the agent to choose $d_{1}=d_{2}$ (the strength of this force depends on the degree of subadditivity). By contrast, there is no such discontinuity under RM. Therefore, if the data exhibit significant clustering on equal deductiblesbeyond that implied by having similar loss probabilities and base premia-the data will tend to select RDPW with a subadditive $\pi(\mu)$.

\section{B. Simulated Field Data}

To buttress the intuition developed in Section IIA, we perform simulations in which we assume first that the RM model is the data generating process (DGP) and then that the RDPW model is the DGP. We calibrate both models according to the data and estimates reported in Barseghyan et al. (forthcoming). To lay bare the different predictions of the models, we consider a stylized environment with two loss events and four deductible options. The set of deductible options is $\{\$ 100, \$ 250, \$ 500, \$ 1,000\}$.

In each simulation, we consider a population of 5,000 agents with homogeneous preferences. Heterogeneity in deductible choices arises from three sources: heterogeneity in loss probabilities $\boldsymbol{\mu}=\left(\mu_{1}, \mu_{2}\right)$, heterogeneity in base premia $\mathbf{q}=\left(q_{1}, q_{2}\right)$, and McFadden choice noise $\varepsilon$. For each loss event $n$, we assume: (i) $\ln \mu_{n}$ is i.i.d. $N(-2.6,0.4)$, yielding a mean of 9.1 percent; (ii) $\ln q_{n}$ is i.i.d. $N(5.80,0.75)$, yielding a mean of $\$ 480$; (iii) $\varepsilon$ is i.i.d. type 1 extreme value with scale parameter $\sigma$, where $\sigma$ is chosen such that approximately 20 percent of choices are altered by noise; and (iv) premiums are given by $p_{n}(\$ 1,000)=(0.8) q_{n}, p_{n}(\$ 500)=q_{n}$, $p_{n}(\$ 250)=(1.2) q_{n}$, and $p_{n}(\$ 100)=(1.5) q_{n}$.

In addition, we assume $\psi(\mu)=\pi(\mu)=0.061+$ $1.186 \mu-2.634 \mu^{2}$ (which is subadditive on the relevant range). We further assume CRRA utility, $u(z)=z^{1-\rho} /(1-\rho)$, and we fix initial wealth at $w=\$ 33,000$ (2010 US per capita disposable income). The coefficient $\rho$ differs
Panel A. RM model

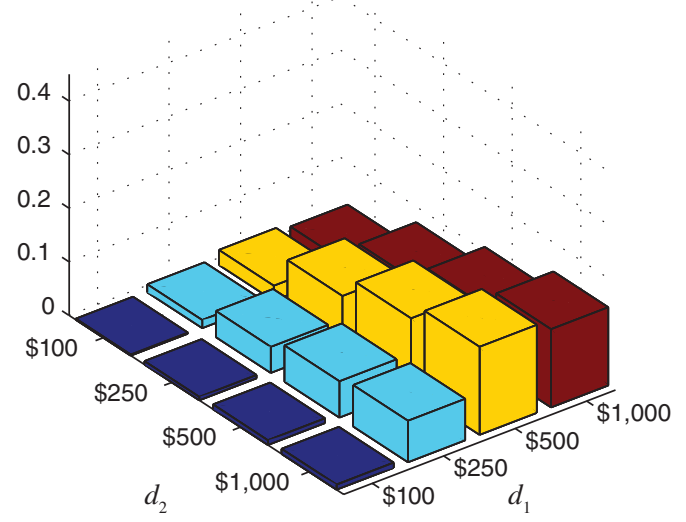

Panel B. RDPW model

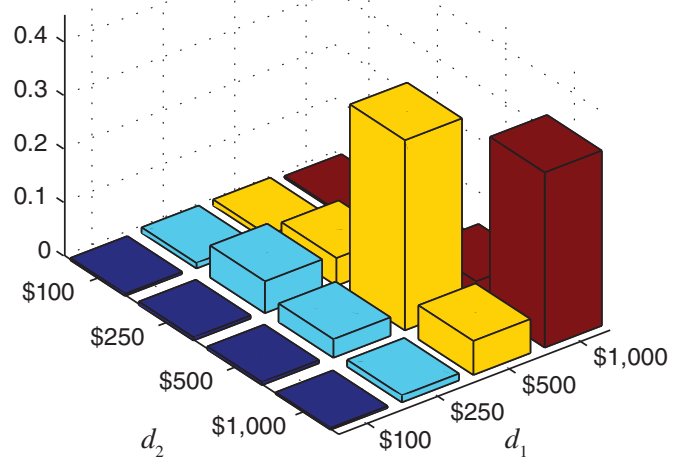

Figure 2. Histograms of Simulated Choices

by simulation. In the RM simulation $\rho=17.0$, whereas in the RDPW simulation $\rho=41.3$. These values are chosen so the average agent in each simulation has a similar "overall" aversion to risk. Specifically, they ensure that an agent with the mean loss probability vector, $\overline{\boldsymbol{\mu}}=(0.091,0.091)$, and the mean base premium vector, $\overline{\mathbf{q}}=(\$ 480, \$ 480)$, is indifferent (absent choice noise) between $\mathbf{d}=(\$ 500$, $\$ 500)$ and $\mathbf{d}=(\$ 1,000, \$ 1,000)$, whether the agent has RM or RDPW preferences.

Figure 2 compares the distributions of simulated deductible choices under RM and RDPW. It clearly reflects the intuition developed in Section IIA. Under RM, the cross-event deductible choices are nearly uncorrelatedthe correlation between $d_{1}$ and $d_{2}$ is 0.05 . Under 
Panel A. Estimated $\psi(\mu)$

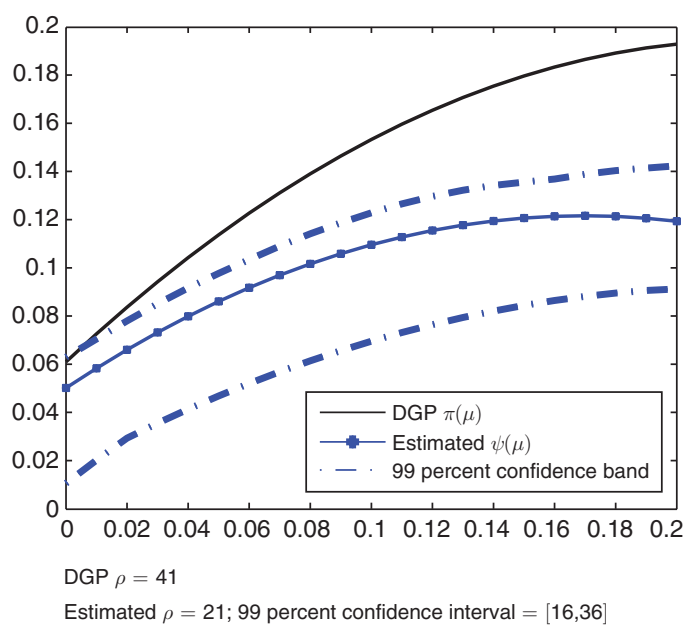

Panel B. Simulated choices

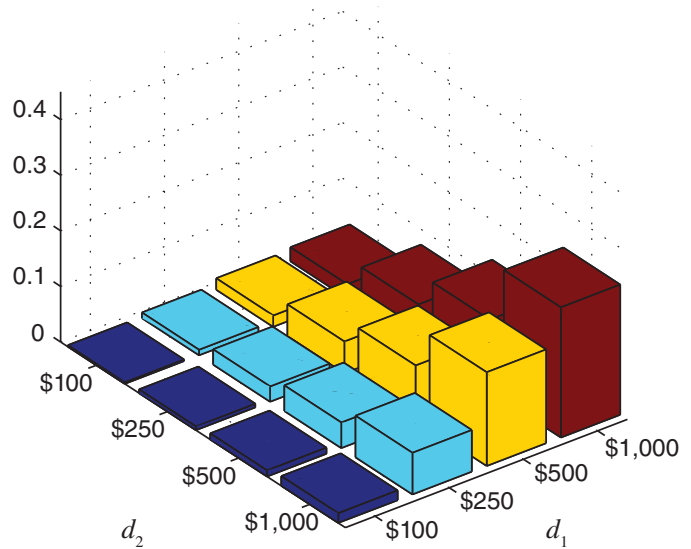

Figure 3. Best Fit of the RM Model

RDPW, by contrast, the cross-event deductible choices are heavily concentrated along the main diagonal (on which $d_{1}=d_{2}$ ), and the correlation between $d_{1}$ and $d_{2}$ is 0.70 .

\section{Toward Distinguishing RDPW from RM}

The simulations in Section IIB clearly demonstrate that a population of RDPW agents generates a different pattern of deductible choices than a population of RM agents with comparable preferences. To truly distinguish RDPW from RM, however, one must rule out the possibility that a population of RM agents with different preferences-i.e., different $\psi(\cdot)$, $\rho$, and $\sigma$-could generate a pattern of deductible choices that is similar to the one generated by a population of RDPW agents. As a first pass, we estimate the best fit of the RM model to the simulated data from the RDPW model. In particular, we specify $\psi(\mu)=a+b \mu+c \mu^{2}$ and then estimate the model parameters- $a, b, c, \rho, \sigma-$ by maximum likelihood.

Figure 3 presents the estimated $\psi(\mu)$ and the distribution of simulated deductible choices under the best fit of the RM model. Panel A demonstrates that the best fit $\psi(\mu)$ and $\rho$ are clearly different from those used to generate Figure 2, panel A. Panel B clearly illustrates that the best fit of the RM model cannot mimic the RDPW model. Indeed, a comparison of Figure 2, panel $\mathrm{B}$ and Figure 3, panel B reveals that, unlike the RDPW simulated data, the best fit of the RM model still has the key distinguishing feature that the cross-event deductible choices are nearly uncorrelated-the correlation between $d_{1}$ and $d_{2}$ now is 0.03 .

\section{REFERENCES}

Barberis, Nicholas. 2013. "The Psychology of Tail Events: Progress and Challenges." American Economic Review 103 (3): http://dx.doi. org/10.1257aer.103.3.XX.

Barseghyan, Levon, Francesca Molinari, Ted O'Donoghue, and Joshua C. Teitelbaum. Forthcoming. "The Nature of Risk Preferences: Evidence from Insurance Choices." American Economic Review.

-Cohen, Alma, and Liran Einav. 2007. "Estimating Risk Preferences from Deductible Choice." American Economic Review 97 (3): 745-88.

-Fehr-Duda, Helga, and Thomas Epper. 2012. "Probability and Risk: Foundations and Economic Implications of Probability-Dependent Risk Preferences." Annual Review of Economics 4 (1): 567-93.

- Jullien, Bruno, and Bernard Salanié. 2000. "Estimating Preferences under Risk: The Case of Racetrack Bettors." Journal of Political Economy 108 (3): 503-30.

- Kahneman, Daniel, and Amos Tversky. 1979. "Prospect Theory: An Analysis of Decision under Risk." Econometrica 47 (2): 263-91.

-Prelec, Drăzen. 1998. "The Probability Weighting Function.” Econometrica 66 (3): 497-527. 
Quiggin, John. 1982. "A Theory of Anticipated Utility." Journal of Economic Behavior and Organization 3 (4): 323-43.

- Snowberg, Erik, and Justin Wolfers. 2010. "Explaining the Favorite-Long Shot Bias: Is It Risk-Love or Misperceptions?" Journal of Political Economy 118 (4): 723-46.
-Sydnor, Justin. 2010. "(Over)insuring Modest Risks." American Economic Journal: Applied Economics 2 (4): 177-99.

- Tversky, Amos, and Daniel Kahneman. 1992. "Advances in Prospect Theory: Cumulative Representation of Uncertainty." Journal of Risk and Uncertainty 5 (4): 297-323. 